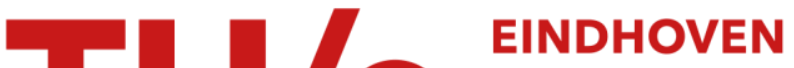

\section{Scan-to-graph: Semantic enrichment of existing building geometry}

\section{Citation for published version (APA):}

Werbrouck, J., Pauwels, P., Bonduel, M., Beetz, J., \& Bekers, W. (2020). Scan-to-graph: Semantic enrichment of existing building geometry. Automation in Construction, 119, [103286].

https://doi.org/10.1016/j.autcon.2020.103286

\section{Document license:}

TAVERNE

DOI:

10.1016/j.autcon.2020.103286

Document status and date:

Published: 01/11/2020

\section{Document Version:}

Publisher's PDF, also known as Version of Record (includes final page, issue and volume numbers)

\section{Please check the document version of this publication:}

- A submitted manuscript is the version of the article upon submission and before peer-review. There can be important differences between the submitted version and the official published version of record. People interested in the research are advised to contact the author for the final version of the publication, or visit the $\mathrm{DOI}$ to the publisher's website.

- The final author version and the galley proof are versions of the publication after peer review.

- The final published version features the final layout of the paper including the volume, issue and page numbers.

Link to publication

\section{General rights}

Copyright and moral rights for the publications made accessible in the public portal are retained by the authors and/or other copyright owners and it is a condition of accessing publications that users recognise and abide by the legal requirements associated with these rights.

- Users may download and print one copy of any publication from the public portal for the purpose of private study or research.

- You may not further distribute the material or use it for any profit-making activity or commercial gain

- You may freely distribute the URL identifying the publication in the public portal.

If the publication is distributed under the terms of Article $25 \mathrm{fa}$ of the Dutch Copyright Act, indicated by the "Taverne" license above, please follow below link for the End User Agreement:

www.tue.nl/taverne

Take down policy

If you believe that this document breaches copyright please contact us at:

openaccess@tue.nl

providing details and we will investigate your claim. 


\title{
Scan-to-graph: Semantic enrichment of existing building geometry
}

\author{
Jeroen Werbrouck $^{\mathrm{a}, \mathrm{d}, *}$, Pieter Pauwels ${ }^{\mathrm{a}, \mathrm{b}}$, Mathias Bonduel ${ }^{\mathrm{c}}$, Jakob Beetz ${ }^{\mathrm{d}}$, Willem Bekers ${ }^{\mathrm{a}}$ \\ ${ }^{a}$ Department of Architecture and Urban Planning, Ghent University, Ghent, Belgium \\ ${ }^{\mathrm{b}}$ Department of the Built Environment, TU Eindhoven, Eindhoven, the Netherlands \\ ${ }^{\mathrm{c}}$ Department of Civil Engineering, Technology Cluster Construction, KU Leuven, Ghent, Belgium \\ ${ }^{\mathrm{d}}$ Faculty of Architecture, RWTH Aachen University, Aachen, Germany
}

\section{A R T I C L E I N F O}

\section{Keywords:}

Linked Data

Existing buildings

Scan-to-BIM

Building Information Modelling

\begin{abstract}
A B S T R A C T
Building Information Modelling (BIM) has changed the way in which buildings are conceived, planned and executed. Apart from their frequent use for as-planned buildings, BIM authoring tools have now been adopted for a number of years for digitising existing buildings as well, mostly by performing a 'scan-to-BIM' process: the creation of a BIM model, primarily based on point clouds. However, some inherent characteristics of existing buildings are complicating such a process: uncertainties, geometric irregularities, classification of heritage building components, linking sources about the real-world asset and an interdisciplinarity that may go beyond traditional Architecture, Engineering and Construction (AEC) topics (e.g. heritage, Facility Management, sensor data and damage assessment). In this paper, a framework called 'scan-to-graph' (STG) is proposed to integrate the concepts of scan-to-BIM with Semantic Web technologies, as these could provide answers to the abovementioned challenges, most notably on documentation of uncertainties, sources and modelling decisions, element classification and cross-discipline information linking. In order to test the STG concept, a use case was developed where the Audience Room of the Gravensteen castle in Ghent was reconstructed from point clouds, semantically enriched and stored as an RDF graph. The resulting graph contains multiple interlinked geometry types, metadata about the reconstruction process and the sources and allows to unambiguously refer to other contextual data on the Web.
\end{abstract}

\section{Introduction}

\subsection{BIM for existing buildings}

The advent of Building Information Modelling (BIM) has brought a revolution in the way in which a construction project is designed, analysed and managed. BIM addresses the need for a hub that contains architectural, engineering and 'Mechanical, Electrical, Plumbing' (MEP) information, thereby allowing information exchange between the various stakeholders that are involved in such a project. As a result, these disciplines can work together in a much more streamlined way than before $[1,2]$.

The potential to manage existing buildings ('as-is BIM', [3]) is less common in practice than the typical use of BIM for planning a new building ('as-planned'). Hence, it is often not sufficiently covered by many software applications. Facility Management (FM), renovation projects, circular economy, building dismantling and management of cultural heritage are all topics that can benefit from semantically rich, digital representations of existing buildings. However, creating an as-is BIM model differs significantly from creating an as-planned model. Since a BIM model of an existing building is based on a real asset, collecting information about this asset is a logical first step. Typically, starting points for documenting an existing building are 'as-planned' documents such as plans and sections. Yet these are often incomplete, out of date or sometimes even missing. Furthermore, the way in which a building is eventually built may differ substantially from the original design envisaged by the architect. Therefore, one needs to conduct onsite surveys on the real asset to gather information about its actual state.

Currently, the most efficient way to conduct those (geometric) surveys is by making use of laser scanning or photogrammetry, both resulting in a point cloud, which can be used as an underlay to create an as-is model. Therefore, the process to make a BIM model based on such point clouds is mostly called 'scan-to-BIM' [3]. The usage of BIM tools for existing buildings is expected to become a game-changer for digital management of existing buildings, similar to what it was in the context

\footnotetext{
* Corresponding author at: Ghent University, Department of Architecture and Urban Planning, J. Plateaustraat 22, 9000 Ghent, Belgium.

E-mail addresses: jeroen.werbrouck@ugent.be (J. Werbrouck), pipauwel.pauwels@ugent.be (P. Pauwels), mathias.bonduel@kuleuven.be (M. Bonduel), beetz@caad.arch.rwth-aachen.de (J. Beetz), willem.bekers@ugent.be (W. Bekers).
} 
of new buildings $[4,5]$.

\subsection{Problem analysis}

In order to become a more frequently applied process, the concept of scan-to-BIM first needs to overcome several obstacles however, as outlined by [3]:

1. The costly, time-intensive and error-prone as-built modelling, due to imperfect real-world geometry.

2. The lack of an incentive to keep the as-is BIM model up-to-date.

3. The lack of an efficient way to deal with uncertain data (occlusions, internal structure, etc.), sources and metadata (e.g. survey equipment).

The first obstacle relates to the very time-consuming process of creating a semantically rich geometric model: a human modeller is always needed to fill the semantic and spatial gaps in the used sources. Currently, no fully automatic process exists to create a BIM model from point clouds, although this is an active research domain (Section 2.2). Nowadays, both geometric modelling and semantic classification are largely done manually or semi-automatically, using plugins for BIM authoring tools. This challenge further relates to the nature of current BIM and CAD authoring tools: they are optimised for planning new buildings, maintaining a rather orthogonal fashion and a strong preference for platonic bodies without irregularities. Consequently, modelling in a typical BIM authoring tool requires higher human effort, as these are mostly not optimised for dealing with 'out-of-plumb' geometries of existing buildings. Semi-automatic algorithms [6-8] and commercial plugins (e.g. FARO PointSense or Leica CloudWorx) are simplifying this process to a certain extent. The quality of the result, however, strongly depends on the specific case. Manual refinement is in most cases indispensable $[3,9,10]$.

The second obstacle is the difficulty for adjacent disciplines to become more BIM-compliant, despite their relevance in the building life cycle. For example, efforts are made to integrate Facility Management (FM) with more construction-focused disciplines (Architecture, Structure, MEP) [5,11,12]. The present focus of BIM tools on constructing new buildings enables a streamlined workflow for these cases, but at the same time, this results in a closed ecosystem impeding a more thorough integration of FM, sensor, GIS or historical data, and renders difficulties in linking multiple geometric representations of the building to the overall model, such as point clouds, imagery, meshes and other 3D-reconstructions of building elements. According to [3], this is one of the reasons why as-is BIM models are not kept up to date. As a result, the data quickly becomes outdated and does no longer reflect the actual state of the building.

The last obstacle is, again, concerned with the industry's current focus on new buildings, which limits the possibilities to efficiently add specific metadata to the project, such as the sources on which a (geometric or semantic) reconstruction was based, the assumptions that were made when information was not available or uncertain (e.g. when an object is partly contained in a wall) or the survey equipment that was used. Therefore, a reliable method is needed for documenting the sources that were used to digitally reconstruct a particular building element and assumptions made during the reconstruction process.

It is important to keep track of this information, for it would be quite misleading if assumed object geometries or attributes (e.g. the substituent layers of a wall) were modelled as if they were known for sure. For example, engineers analysing building performance or structural integrity need to know which data is uncertain and on which sources this data is based. This is crucial when determining properties that can be used for simulating specific scenarios.

An additional challenge is the fact that current BIM authoring tools or neutral exchange formats such as IFC (Industry Foundation Classes) provide only a limited amount of building component classes. This causes problems when non-contemporary elements, uncommon in newly built structures, have to be classified.

\subsection{Research questions}

Meanwhile, independent from BIM and existing buildings, a global trend emerges where researchers from various disciplines interlink their data using web technologies. This interdisciplinary 'Web of Linked Data' or 'Semantic Web' [13] is built upon the principles of the classic world wide web, focusing on describing data in an open and interdisciplinary fashion, separated from the software applications that use this data. Some disciplines are already firmly rooted in the Semantic Web, such as the geospatial domain, government, life sciences, etc. There is a trend to translate the current BIM practices of the AEC industry into the principles of Linked Data [14-16], as such technologies provide various advantages, predominantly regarding interoperability, cross-discipline linking and logical inference [17]. The main research question in this article is therefore whether Linked Data technologies can provide a basis for an approach that addresses the above-mentioned challenges. In order to answer this, we consider the following sub-questions:

1. Can a workflow be devised to create a Linked Data-based digital model of an existing building, and what could this workflow look like?

2. Can heterogeneous data sources be unambiguously and semantically linked to form an integrated model of a built asset?

3. Can modelling assumptions and inherent uncertainties be documented in this integrated model?

4. Can this model facilitate integration of information from the many disciplines involved with existing buildings?

With Linked Data graphs as the main technology, we will review and address these questions in this paper. In order to do this, we will broaden the scan-to-BIM concept to 'scan-to-graph' (STG). Vital to the creation of a reliable as-is digital model, the integration of sources for the reconstruction, metadata and assumptions is considered an essential part of this concept. Due to the flexibility of Linked Data technologies, existing classification schemes can be used and when necessary extended to incorporate non-contemporary elements, and the primary building data can be linked to relevant data from adjacent disciplines such as GIS (Geographic Information Systems), Facility Management and historical data. In this way, the as-is reconstruction becomes a multi-purpose model from which, depending on the use case, different information can be extracted for use and manipulation in dedicated software applications.

\subsection{Article structure}

In the following section, a brief outline is given of the most common remote sensing techniques for generating point clouds for a scan-to-BIM process (Section 2.1), along with a brief discussion on the current research state regarding scan-to-BIM (Section 2.2). Then, an introduction to Linked Data, the Semantic Web (Section 2.3) and its current state of the art within the construction industry (Sections 2.4 and 2.5) will provide the background for the scan-to-graph workflow and corresponding vocabulary in Section 3. Section 4 discusses a prototype plugin for the CAD application Rhinoceros 6 (McNeel), which supports the direct creation of an RDF graph of the existing building. The process is then demonstrated with the modelling of a case study, the Audience Room in the Gravensteen Castle in Ghent, Belgium (Section 5). The paper concludes with Section 6, discussing general findings and future work. 


\section{Background and related work}

\subsection{On-site surveying}

Whenever coping with existing buildings - whether in case of heritage management, renovation or education - the access to reliable information is of the utmost importance. Although original plans and sections, if available, mostly provide a decent idea of a building 'asplanned', they do not necessarily provide information about its actual 'as-is' or 'as-built' state. To obtain the as-is building geometry, realworld surveys are often inevitable.

A number of survey techniques allow retrieving spatial information about a building structure. These techniques can be divided into two large groups: non-intrusive techniques and intrusive techniques. Nowadays, the most efficient methods are non-intrusive: they are quick, reliable and touching fragile or unreachable surfaces is not necessary. Advanced non-intrusive technologies, such as total stations, laser scanners and photogrammetric reconstructions are often referred to as 'remote sensing' techniques, since they do not make physical contact with the object being measured [3].

A total station measures the 3D position of discrete points, which can be used as control points to digitally reconstruct the building geometry. Laser scanners and photogrammetric surveys, in contrast, return an immense amount of data in the form of a point cloud: a digital, spatial representation of the object, containing measurements of thousands or even millions of points. Therefore, laser scanning and photogrammetry are called High Definition Surveying (HDS) techniques.

Although laser scanning and photogrammetry may seem competing survey methods, they can complement each other in many cases $[5,18,19]$, as different acquisition methods are used and the generated point clouds do not have exactly the same features regarding resolution and accuracy. For example, depending on the accessibility of the location to capture, one could choose to switch between laser scanning and photogrammetry. Further reasons to combine both methods are edge detection for surface reconstruction - which is more feasible with photographs - or adding colour to laser scanned point clouds by 'plotting' the colours from a photograph onto the point cloud. This feature is often directly integrated in modern laser scanners.

Point clouds are de facto the most complete sources that are currently available for geometric digitisation of real-world objects, describing the visible parts of a structure in high detail. However, they are essentially just a collection of points containing spatial coordinates, sometimes also incorporating RGB (colouring) values, normals and an intensity value. They do not provide any information about the nature of the object being scanned, its boundaries or the membership of a larger building object. Although a point cloud can provide detailed geometric information, it is very poor from a semantic point of view. Therefore, point clouds often serve as a basis for more storage-friendly and semantically rich models, such as BIM models.

\subsection{Scan-to-BIM}

More recently, considerable progress has been made in the conversion from point clouds to BIM models (scan-to-BIM). A bibliographic analysis by [5] reveals growing interest in the field of BIM for existing buildings and its use cases from 2014 on. Multiple research projects are focusing on refinement of the scan-to-BIM process, either oriented towards holistic procedures or towards specific subtasks such as point cloud alignment, automated segmentation and classification, topological recognition, geometric fitting, control mechanisms etc.

In the field of automated segmentation, [20] use octree-based region growing and conditional random field clustering to segment a point cloud into meaningful parts. [21] use local convex and concave properties to segment the point cloud's structural parts and reconstruct a 3D model based on this segmentation. Classification of segmented regions in point clouds is increasingly done by making use of machine learning and computer vision algorithms [8]. Worth mentioning as an overall approach is the DURAARK software prototype [7], an opensource prototype for the recognition of IfcWalls, IfcDoors and IfcWindows in point clouds. It was constructed in context of the DURAARK European project ${ }^{1}$ (DURAble ARchitectural Knowledge), which focused on the preservation and archiving of digital building data. An extension of this research towards a more flexible and encompassing methodology was recently described in [22]. An overal review of performance evaluation of machine learning algorithms for object recognition in point clouds is discussed in [23].

A general approach that structures the entire process from data gathering to design adaptations was introduced by [24], called BIMification. It describes and structures the different stages in a scan-to-BIM process in two main phases: 'Anamnesis' and 'Diagnosis'. A third phase, 'Therapy' is identified for the activities that make use of the reconstructed BIM model (such as retrofit planning).

Because the interest in BIM for existing buildings is rather new, there is not much standardisation related to this domain. A mapping of current standards and formats and their potential for existing buildings has been done by [4]. In the meantime, the USIBD Level of Accuracy (LOA) [25] gains traction as a standardised metric for the deviation between a point cloud and a geometric reconstruction. A more detailed approach for a standardised geometric quality assessment between point cloud and digital reconstruction of building components has been outlined in [26].

It needs to be stressed that the approach proposed in this paper may follow from each of the above-mentioned scan-to-BIM methodologies, since recognition and classification methods are independent of the data structure that is chosen to store the information about the built asset. The scan-to-graph process should thus not be seen as a competing strategy with scan-to-BIM algorithms, but rather as a data structure that can be applied on the output of such scan-to-BIM algorithms, in order to make it more interoperable with other disciplines and keep track of the reconstruction process.

\subsection{Semantic web technologies}

We tend to move towards an ever more automatised world, with information sources and stores connected with a web of digital agents. Additionally, more and more work is meant to be done by computers and algorithms ${ }^{2}$ [13]. Information exchange is one of the core concepts of the Web, therefore, it is essential to have a standardised communication framework enabling all actors to 'speak' with one another, which is the purpose of the Resource Description Framework (RDF). ${ }^{3}$ $\mathrm{RDF}$ allows to express and link individual statements with one another and is currently the main W3C (World Wide Web Consortium) standard for representing, managing and connecting fine-grained data via Uniform Resource Identifiers (URIs).

In RDF, all information is represented by so-called triples, which form an RDF graph. RDF triples, based on URIs, can be compared with very basic sentences, containing 'resources': a 'subject', an 'object' and something that establishes a directed relationship between these two, referred to as the 'predicate'. When several resources together form a conceptual schema related to a specific domain, an 'ontology' or 'vocabulary ${ }^{4}$ is defined using RDFS ${ }^{5}$ or OWL. ${ }^{6}$

Ontologies do not only organise concepts on a specific domain, but also aid in negotiating between contradictory information on the web

\footnotetext{
${ }^{1}$ http://duraark.eu/.

2 https://www.w3.org/standards/semanticweb/.

${ }^{3}$ https://www.w3.org/TR/rdf-schema/.

${ }^{4}$ https://www.w3.org/standards/semanticweb/ontology.

5 https://www.w3.org/TR/rdf-schema/.

${ }^{6}$ https://www.w3.org/OWL/.
} 
and the process of making implicit information explicit through analysis of existing relationships and definitions in a graph, called 'inference'. ${ }^{7}$ A distinction can be made between resources that refer to concepts and are defined in ontologies, and resources that represent specific instances or data. The terms TBox and ABox refer to this dichotomy: TBox ('Terminological') refers to the group of triples defining classes and properties, while ABox ('Assertion') refers to the group of triples containing nodes that are instances of domain specific classes.

The definitions in an ontology are identified with unique URIs and typically share a common namespace. For example, all the definitions in the Building Topology Ontology ${ }^{8}$ [16] are defined in the BOT namespace https://w3id.org/bot\#. Namespaces can be replaced with prefixes in the Turtle (TTL) syntax for RDF. ${ }^{9}$ Prefixes used in the remainder of this paper are listed in Listing 1.

Listing 1. Namespaces used in this publication.
Table 1

Three triples define the relationship between a specific wall and door (tabular form).

\begin{tabular}{lll}
\hline Subject & Predicate & Object \\
\hline inst:wall1 & rdf:type & beo:Wall-SOLIDWALL. \\
inst:door1 & rdf:type & beo:Door-DOOR. \\
inst:wall1 & bot:hasSubElement & inst:door1. \\
\hline
\end{tabular}

2. Linking of various domains: BIM, product data, GIS, FM, heritage, etc.

3. Logical inference, querying and proofs to check a model against regulations, geometric collisions etc.

The conversion of the EXPRESS-based IFC schemas ${ }^{11}$ into a Linked

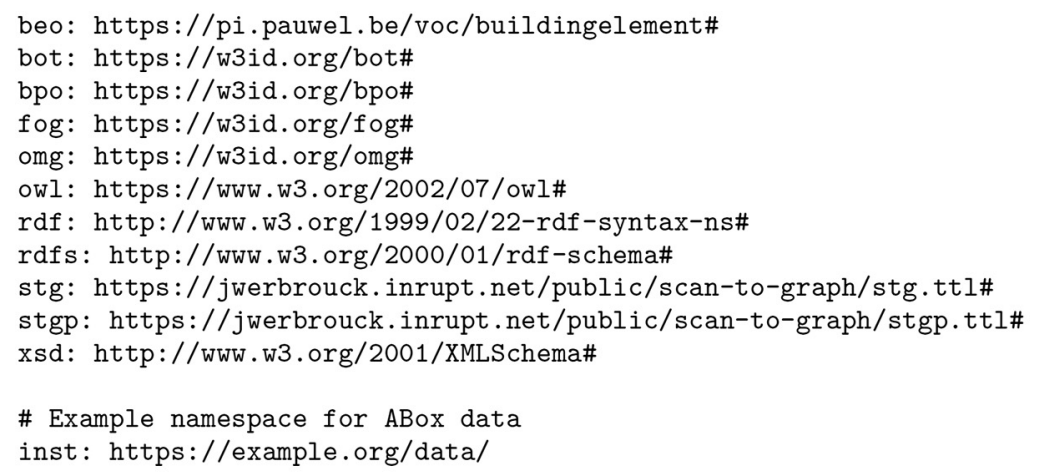

Although a set of triples can be represented in tabular form (Table 1), they are frequently visualised in the form of a directed graph. Nodes in the graph represent subjects and objects, the graph edges represent the predicates, pointing from subject to object. Due to this graph nature, RDF provides a flexible way to store and query data.

The SPARQL Protocol and RDF Query Language (SPARQL) ${ }^{10}$ lends itself as a powerful language, not only to query an RDF graph, but also to manipulate it. In a building context, SPARQL can play an important role in querying distributed databases for new information. For example, it can be used to generate partial models or for retrieving very specific building data (e.g. 'return windows from a specific manufacturer and with specific U-value, that face the northern side of a building').

\subsection{Ontologies for the built environment}

The use of Semantic Web technologies and Linked Data specifically oriented towards the construction industry has gained serious interest over the last decade. However, in practice, services that make use of these technologies remain scarce, due to a large implementation threshold. Nevertheless, a Linked Data-driven BIM paradigm can mean a serious improvement compared to current practice. [17] assembled an extensive literature overview on this topic, and defined three large use cases:

1. Interoperability of data models and representations.

\footnotetext{
${ }^{7}$ https://www.w3.org/standards/semanticweb/inference.

8 https://w3c-lbd-cg.github.io/bot/.

${ }^{9}$ https://www.w3.org/TR/turtle/.

10 https://www.w3.org/TR/sparql11-query/.
}

Data equivalent $[14,15]$ marked an important step for further research on Linked Data. It resulted in a backwards compatible OWL equivalent for each IFC EXPRESS schema, namely ifcOWL. The IFC schema, however, is quite complex and includes an enormous amount of classes and properties, which is reflected in the size of the ifcOWL ontology. Due to this complexity, RDF graphs that are based on ifcOWL are difficult to query efficiently. Additionally, it complicates linking schemas from different domains: the larger the ontologies to connect to one another, the more intensive the mapping process. Because of the inherent size and complexity of ifcOWL, current research by the W3C Linked Building Data (LBD) ${ }^{12}$ Community Group focuses on developing small, modular vocabularies that are easier to use and maintain [27], and much lighter compared to ifcOWL graphs [28]. A proof of concept conversion tool between IFC and the LBD-based RDF graphs is publicly available. ${ }^{13}$ Additionally, an implemented approach to use conventional BIM data and LBD graphs in parallel, including synchronisation, has been proposed in $[29,30]$.

For the STG method proposed in Section 3, these lightweight LBD ontologies are used as much as possible. Therefore, some background on some core LBD ontologies is necessary, specifically those concerned with general building topology and building element taxonomy. The root LBD ontology is BOT (Building Topology Ontology) [16], which defines the topological relationships between the (sub)components of a building (Fig. 1). A bot:Building consists of spatial bot:Zone and bot:Element instances. Subclasses of bot:Zone are bot:Site, bot:Building, bot:Storey and bot:Space. Apart from containing other bot:Zone

\footnotetext{
${ }^{11}$ https://technical.buildingsmart.org/standards/ifc/ifc-schemaspecifications/.

12 https://www.w3.org/community/lbd/.

13 https://github.com/jyrkioraskari/IFCtoLBD.
} 


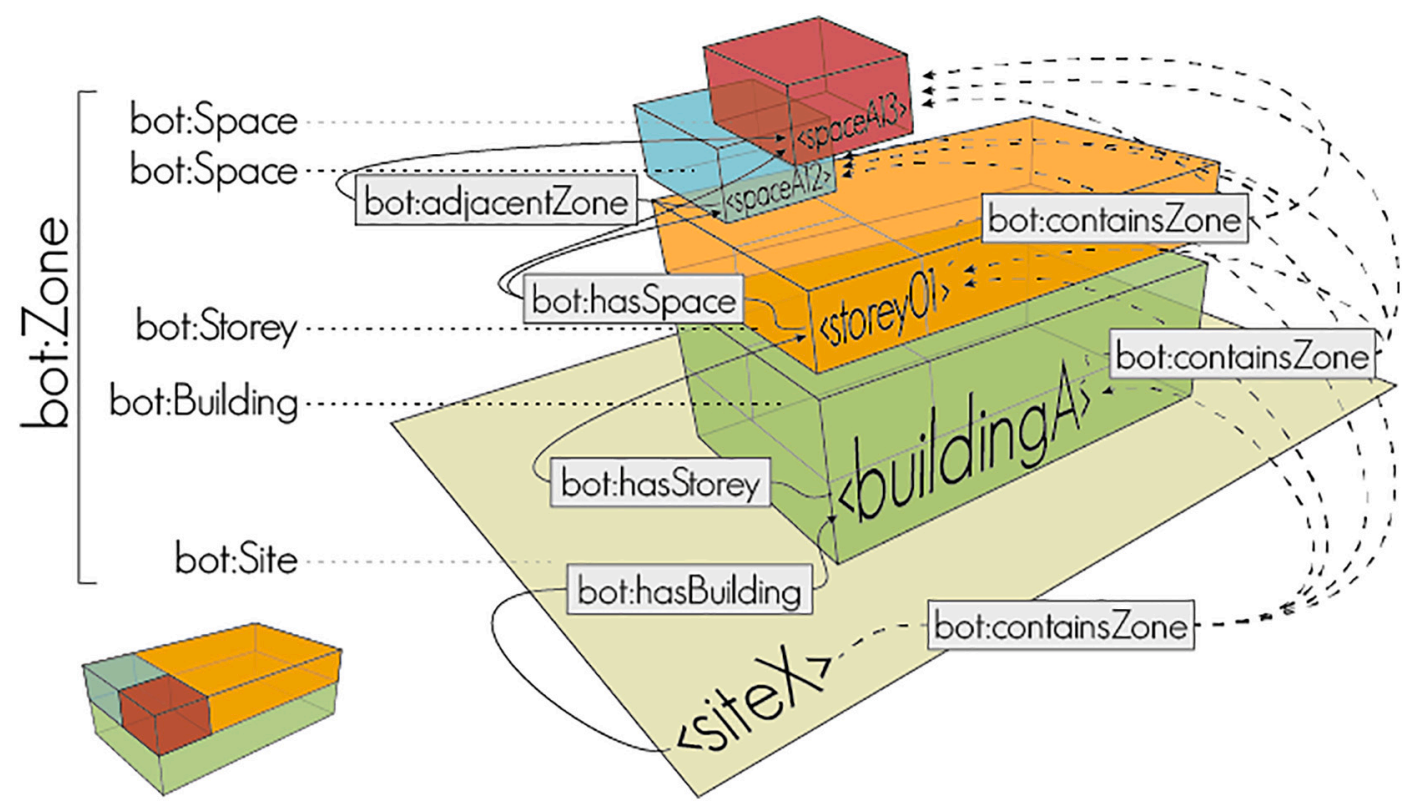

Fig. 1. Topological relationships in the BOT ontology.

(Source: Rasmussen et al. [16]).

instances, a bot:Zone individual can also be adjacent to another one, or intersect it. Each zone can be bounded by physical building elements and can also contain them. Building elements can also host other building elements (e.g. a wall can host a window). A bot:Interface between two zones, two elements or both a zone and an element can be defined. Fig. 1 depicts the spatial relationships between the different subclasses of bot:Zone. BOT also defines one generic building element class, bot:Element, which can be further specified using a preferred product or classification ontology.

As a basis for object classification, the BuildingElement Ontology ${ }^{14}$ defines different types of building elements and furniture, based on the eponymous subtree within the IFC schema. However, its purpose is not to cover the entire range of all possible building products, which would be impossible. Rather, it defines the most common ones and allows for custom extensions. For instance, in the case of existing buildings and heritage, which often have very specific, non-contemporary elements, it is possible to create custom classes as a custom extension to the BuildingElement ontology or as a stand-alone new domain ontology. An instance can thereby be assigned to multiple classes, for example an element can be at the same time a beo:Door, a beo:Window and a bot:Element. This in contrast with regular BIM modelling, where exactly one class is defined for each building object. Other classification systems that may be useful in the case of existing buildings are the Getty Art and Architecture Thesaurus (AAT), ${ }^{15}$ WikiData, ${ }^{16}$ GoodRelations, ${ }^{17}$ and the buildingSMART Data Dictionary (bSDD)..$^{18}$

It may be relevant in many use cases to further deconstruct a building element into several constituent parts, which are also to be classified. In this scenario, the Building Product Ontology (BPO) ([31]) is recommended to describe these assemblies (bpo:Assembly). Although detailed relationships between bpo:Component instances and bpo:Assembly instances can be established using BPO, this text will only make use of the general relationship bpo:isPartOf.

\footnotetext{
${ }^{14}$ http://pi.pauwel.be/voc/buildingelement.

15 http://www.getty.edu/research/tools/vocabularies/lod/.

16 https://www.wikidata.org/.

${ }^{17}$ https://www.w3.org/wiki/GoodRelations.

18 https://www. buildingsmart.org/standards/standards-tools-services/datadictionary/.
}

\subsection{Geometry in linked data}

The ability to link various geometric representations to a building element is an important feature enabled by the use of Linked Data. Using dedicated definitions for specific geometric formats, a component can refer to a variety of co-existing geometry representations. However, the more diverse geometry representations a graph references, the more difficult it becomes to keep every linked geometry up to date in case of changes. With existing buildings, this does not affect the files that are considered primary sources such as point clouds, since they will not change anymore. In the case of multiple derived geometries, however, this becomes more problematic, as a change in the geometry in e.g. a Revit model will not automatically update its geometric Rhinoceros equivalent. When BIM data is present in collaborative environments such as web-based repositories or databases hosted by third parties, an update of changed geometry might not be allowed. This challenge is addressed in current research on the use of Linked Data for versioning geometries [32], using the Ontology for Managing Geometry (OMG) [33] and the complementary File Ontology for Geometry formats (FOG) [34]. Both ontologies are used in Section 3 for indicating relationships between sources and geometric reconstruction.

Different methods are available to effectively link various geometric representations in an RDF graph. For large geometric datasets such as point clouds or complex meshes, it is generally recommended to not embed these directly into an RDF graph, since these can easily reach sizes of multiple gigabytes [32]. Instead, these files can be stored in a location outside the RDF graph, and referenced to using URLs (e.g. in an online repository, accessible for different project stakeholders). In the case of smaller-size geometric objects (such as single BREP or NURBS geometries), an alternative option is to implement them as RDF-based geometry descriptions. Different implementations are discussed in $[33,35,36]$. Another method is to embed the content of images or geometry files of widely used geometry formats in RDF literals, e.g. using a plain string (text-based geometry) or a binary-to-text encoding such as Base64 (binary geometry). This allows to include different geometric formats in a rather straightforward way and store geometry and semantics together at one location (the RDF graph). All mentioned approaches to relate geometry to semantics are valid within the STG approach. 


\subsection{Summary}

In this section, a brief introduction to scan-to-BIM and Linked Data was given. Furthermore, some existing conceptual implementations and current challenges of Linked Data for the construction industry were discussed, and existing modular vocabularies that will be used for the outline of the STG method (Section 3) were briefly introduced. Finally, strategies for implementing geometry or binary data into RDF graphs were discussed. In the following section, these topics will be related to one another, in order to propose a Linked Data-based workflow in the context of existing buildings.

\section{The scan-to-graph method}

The modular approach with Linked Data technologies presented at the end of Section 2 opens options for addressing some of the current issues in scan-to-BIM projects. More specifically, a modular Linked Data approach may be well-suited to cope with the challenges of linking multiple sources and metadata, classification of non-contemporary elements and combining various geometric representations that can be read by different software tools. With this holistic approach in mind, we consider a dedicated approach for existing buildings: scan-to-graph (STG).

It should be stressed that the scan-to-graph method is essentially independent from the ontologies used. Depending on the project, different ontologies may be used, since the idea is to reach maximum expressiveness. However, a recommended workflow based on existing ontologies and a dedicated core STG vocabulary will be devised in this paper. These existing ontologies include BOT, OMG, FOG, BuildingElement and BPO (Section 2.4); a non-exhaustive list which can be easily extended with other ontologies, should the need rise within a specific project. Extension options include, amongst others, the Damage Topology Ontology (DOT) ([37]) for indicating damage patterns and the Ontology for Property Management (OPM) $([38,39])$ for linking building objects with properties that change over time. The STG vocabulary includes new definitions as well as extensions (subclasses and subproperties) of existing vocabularies. All properties in the vocabulary are object properties. An overview of classes and properties in the STG vocabulary is given in Appendix A.

Starting from the above-mentioned ontologies, the setup of a minimal semantic model that connects with source material (point clouds, imagery...), reconstructions, metadata and assumptions, is the main topic of this section. A minimal scan-to-graph (STG) vocabulary is devised for this purpose. Section 3.1 provides an example of how the BuildingElement ontology can be extended to incorporate non-contemporary or user-defined elements. In Section 3.2, we discuss how to set up a relationship between a reconstruction and its sources, using the OMG and FOG ontologies. Section 3.3 deals with adding metadata about this relationship. Finally, Section 3.5 associates the STG process within the general workflow of a scan-to-BIM process and Section 3.6 highlights the interdisciplinary gains of separating semantic data and geometries from the applications using this information.

\subsection{Topology and element classification}

Both recommended ontologies for topology (BOT) and classification (BuildingElement) have been described in Section 2.4. The setup of a core graph of the model using BOT is quite straightforward, using the relationships depicted in Fig. 1. When this topological foundation is made, building elements are classified and their relationship with the building topology is indicated. When a building element type is not yet available as an owl:Class in the BuildingElement ontology, an extension can be made by defining a subclass of beo:BuildingElement. Alternatively, an entirely new ontology can be created and used. The vocabulary can also be linked to the URI of the Getty AAT ${ }^{19}$ or Wikidata ${ }^{20}$ page that defines this component type. Such linking also embeds new
Table 2

Example of a custom building component type as an extension to the BuildingElement ontology.

\begin{tabular}{lll}
\hline Subject & Predicate & Object \\
\hline stgp:Capital & $\begin{array}{ll}\text { rdf:type } \\
\text { rdfs:subClassOf }\end{array}$ & $\begin{array}{l}\text { owl:Class; } \\
\text { beo:BuildingElement, } \\
\text { bot:Element; } \\
\text { "Capital"@en, } \\
\end{array}$ \\
& rdfs:label & hapiteel"@nl; \\
& http://vocab.getty.edu/aat/300001662, \\
& https://www.wikidata.org/wiki/Q193893. \\
& &
\end{tabular}

definitions in existing (standardised) taxonomies. Table 2 contains an example which identifies the capital of a column. As a custom prefix, 'stgp' is chosen ('STG Product') for the definition of a new vocabulary.

For a further breakdown of building elements into their constituent parts, use of the BPO ontology is recommended (e.g. through the relationship bpo:isPartOf). Building elements may be decomposed when specific semantics are to be related to a particular sub-element, or to indicate that a part of this element is invisible, e.g. because it is encapsulated by a wall. Since the latter case is an important reason for uncertainties about object dimensions or other properties, a dedicated subproperty of bpo:isPartOf is devised as an STG definition, namely stg:isInternalPartOf (Fig. 2).

\subsection{Relating sources and reconstructions}

In the specific scenario where one resource (e.g. a point cloud) forms the basis for another resource (e.g. a reconstructed volume), which is the case in a scan-to-BIM process, one might want to provide additional information about this relationship. Therefore, we base upon the OMG ontology to link various geometric representations to a semantic object, using the omg:hasGeometry property. The relationship between a reconstructed geometry and a source is expressed using the OMG property omg:isDerivedFromGeometry. As mentioned in the OMG documentation, a geometry may be derived from multiple sources. ${ }^{21}$ Respective file extensions for geometry formats are then expressed using the FOG ontology (Fig. 3).

A source node can be classified as an stg:Source; a reconstruction node as stg:Reconstruction. Both are subclasses (rdfs:subClassOf) stg:Representation. Reconstructions are often based on multiple documents, with varying degrees of influence on the final result. To differentiate between these sources, an stg:Source has subclasses stg:MainSource and stg:AuxiliarySource. An stg:Reconstruction can be linked to these sources by respectively stg:hasMainSource and stg:hasAuxiliarySource, both subproperties (rdfs:subPropertyOf) of stg:hasSource. This also allows other sources such as imagery or textual files, which are not within the scope of the FOG ontology, to be related to the instance node.

A resource may refer to geometric instances contained in a larger file [33]. For example, the geometry linked to a beo:Door instance may be present already in a larger geometry document that is linked to the entire building. This is likely to be the case when an as-is BIM model had been created using existing BIM authoring tools, following a regular scan-to-BIM workflow (3.5), and the model is to be further enriched with metadata and sources using the STG method. The entire BIM model would need to be converted to both one or more geometric representations (using a tool such as IfcConvert $^{22}$ ) and to a semantic, RDF-based part, using an IFC to RDF converter tool $([15,28])$ in order to enhance the model with additional metadata and sources. While the

\footnotetext{
19 http://vocab.getty.edu/.

${ }^{20}$ https://www.wikidata.org.

${ }^{21}$ https://www.projekt-scope.de/ontologies/omg/.

22 http://ifcopenshell.org/ifcconvert.
} 


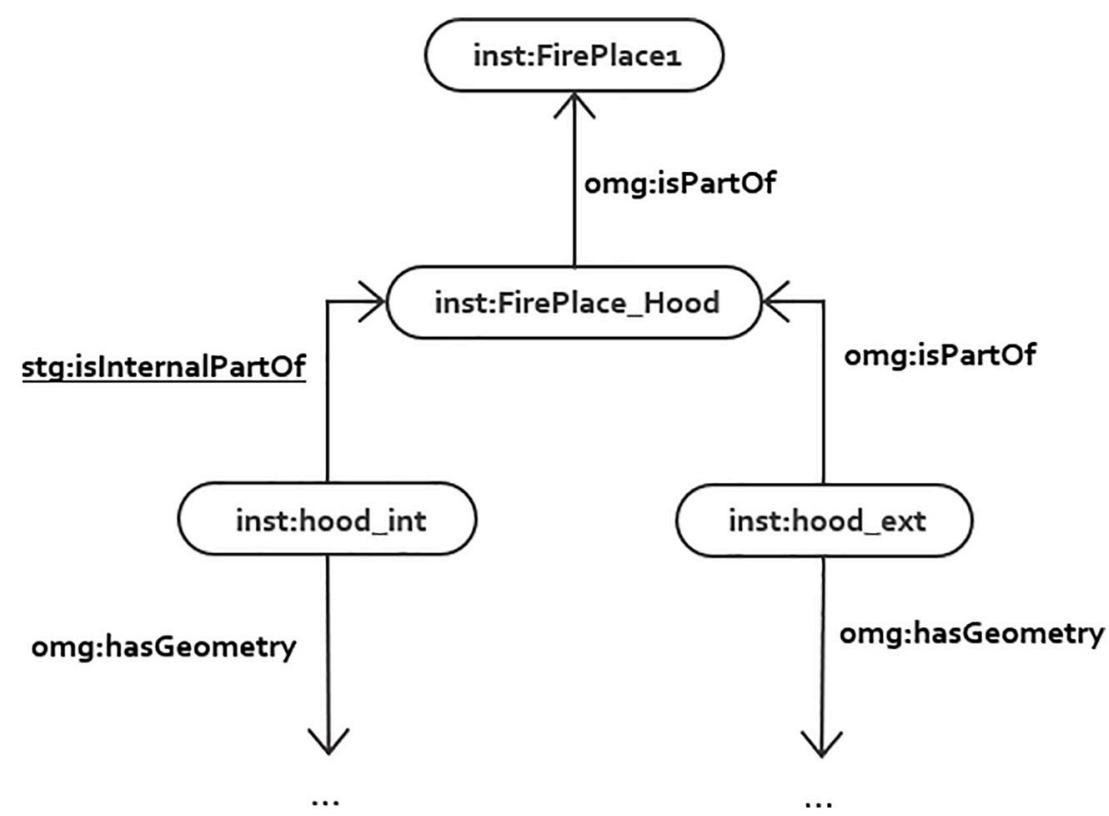

Fig. 2. A geometry that is partly enclosed in another geometry and thus uncertain may be related to the larger geometric instance using stg:isInternalPartOf, a subclass of omg:isPartOf.

semantics expressed in RDF would be fine-grained and linkable, current IFC geometry converters or 3D model files currently do not destructure the geometry into separate elements, as it remains in a larger document, relating to the building elements via GUIDs. In order to connect an element to a geometry contained in such larger file, the contained element may refer to the larger representation using this GUID by which it is identifyable in the larger document, using the OMG and FOG ontology (Fig. 3). The general procedure to extend a regular scan-toBIM process towards scan-to-graph is further discussed in Section 3.5. Note that both individual geometric elements as larger aggregated geometry files may be present in one graph, a scenario used in the plugin described in Section 4 and illustrated through the use case in Section 5 .

\subsection{Relating metadata}

Because specific modelling issues can arise from relying on specific source instances, metadata about a reconstruction process always depends on both source and reconstruction. Apart from the relationship established with stg:hasSource, a metadata resource can be set up that relates to the reconstruction via the stg:hasMetadata property and to the source via stg:basedOn. Via this metadata instantiation, process metadata can be added, such as the LOA resulting from a deviation analysis (stg:hasLOA) or modelling assumptions (stg:hasAssumption).

An stg:Assumption is textually expressed using rdfs:comment, and may be caused by (stg:hasReason), for example, an occlusion (stg:Occlusion) present in the source file. The source file is linked (stg:hasOcclusion) to this occlusion as well, which may be, for example, a bounding box geometry, or an explicit surface that is not visible in the source point cloud. stg:Occlusion is therefore a subclass of omg:Geometry. The occlusion may be resolved in another source, because it might be visible there. This should then be indicated by the property stg:resolvesOcclusion, pointing from the second source towards the occlusion.

\subsection{Synopsys of the STG graph structure}

Combining STG with the vocabularies discussed in Section 2.4, an annotated graph can be constructed for an existing building. This annotated graph, which represents the overall building model, may or may not consist of multiple smaller named graphs, e.g. dedicated to each of the topics discussed in Sections 3.1, 3.2, 3.3. Figs. 2, 3 and 4 depict example ABox graphs as an illustration for the use of the STG vocabulary in a scan-to-graph process. For a Turtle serialisation of the vocabulary, the reader is referred to https://jwerbrouck.inrupt.net/ public/scan-to-graph/stg.ttl.

\subsection{The STG workflow}

It has been stressed in Section 1.3 that the aim of the STG method is not to replace existing Scan-to-BIM technologies, but rather to broaden the workflow so the model can be semantically enriched with additional data. The aim of this section is therefore to relate the STG approach to existing scan-to-BIM workflows, in a scenario where STG is carried out from the beginning on (Fig. 5), and when using existing (semi-automatic) algorithms in existing scan-to-BIM conditions (Fig. 6).

Fig. 5 outlines the different steps carried out when an STG process is carried out from the beginning, i.e. when an RDF graph of the building has been the envisaged end product before starting the process. The 'Source preparation' step includes acquisition of the different sources (point clouds, imagery, plans, textual survey documents) and potential pre-processing of point clouds (e.g. georeferencing, clutter removal, alignment and segmentation). Output of reconstruction and recognition processes is then immediately expressed in RDF and includes semantic links between sources and reconstruction, uncertainties, modelling assumptions, LOA and other metadata. Semantic recognition should both link to the elements and topological instances being classified and to the sources used for this classification (e.g. specific point cloud segments). The end results of the entire process are then stored according to the data types: sources and reconstructed geometries will be stored in a file-based system, while the graph data resides in a triple store, referring to the file-based system via urls. Alternatively, a hybrid solution following the Linked Data Platform (LDP) ${ }^{23}$ specification can be used to store both RDF and non-RDF data.

Fig. 6 illustrates the workflow when existing scan-to-BIM algorithms are used to produce an IFC file. The most defining factor to reach a finegrained semantic model will be the degree to which the outcomes of

\footnotetext{
${ }^{23}$ https://www.w3.org/TR/ldp/.
} 


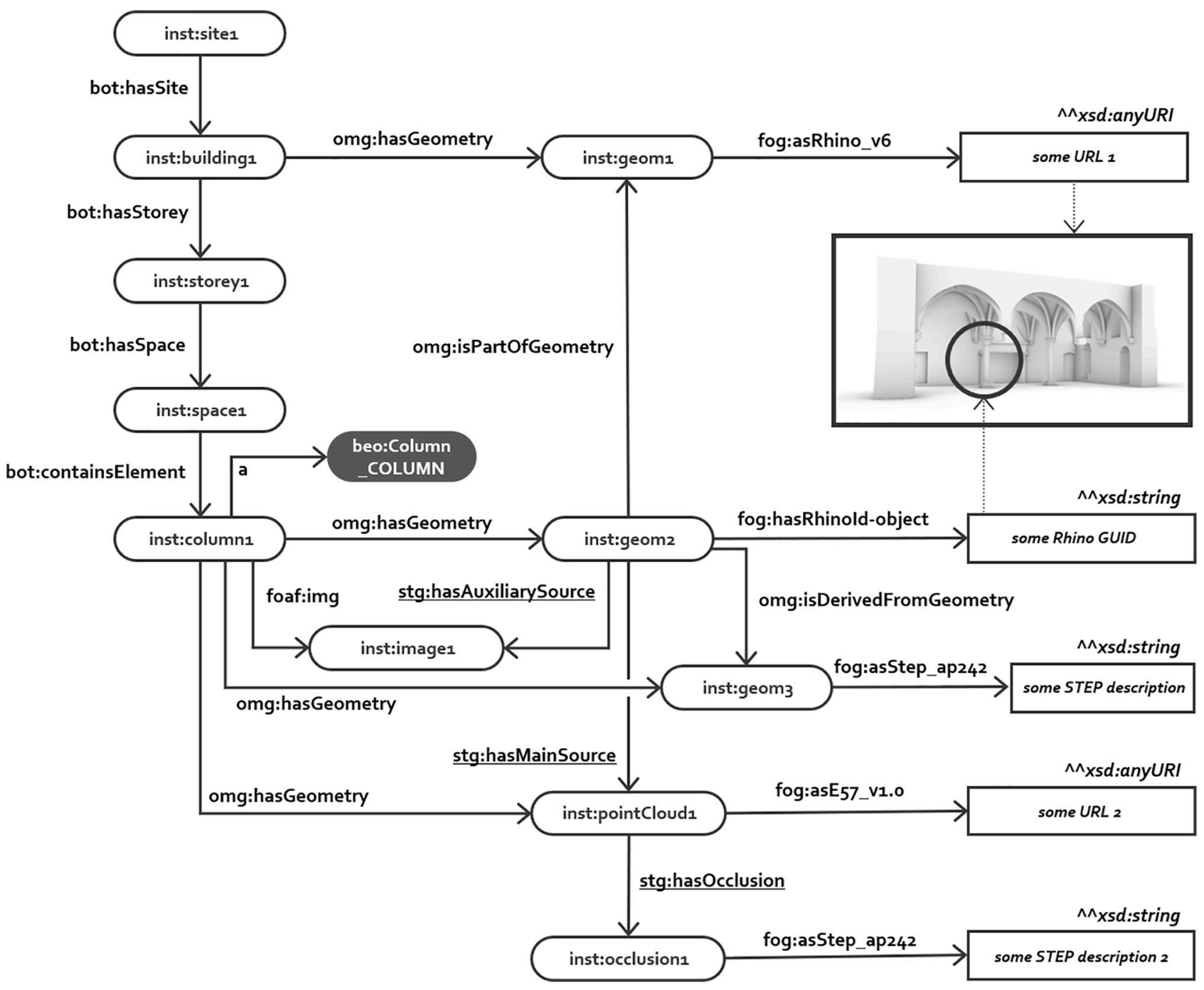

Fig. 3. Linking geometric representations to a semantic element.

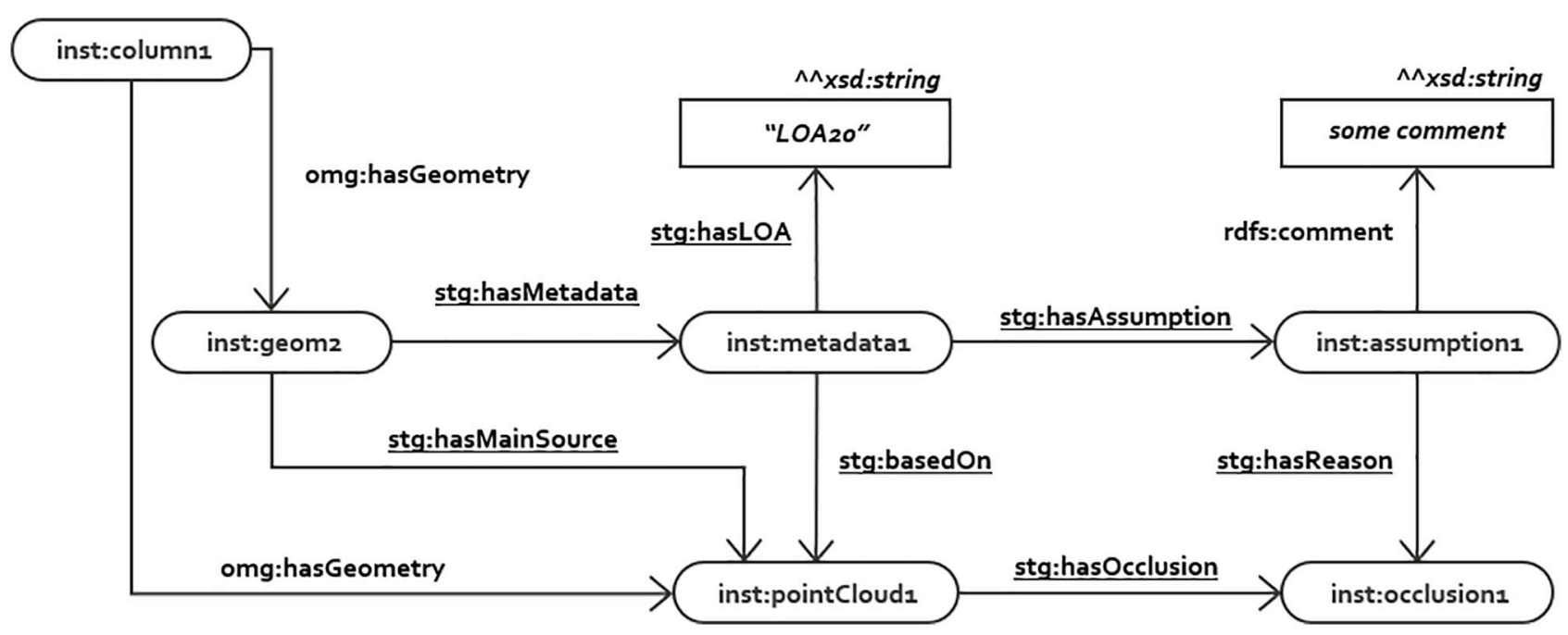

Fig. 4. Linking metadata about the reconstruction process to a semantic element.

geometric reconstructions and semantic recognition processes are still related to the sources used to get to these outcomes; and the documentation of assumptions made during the process. This depends on the algorithm used: is there, apart from the main BIM (IFC) model outcome, a log file that keeps track of the sources that were used? For instance, for a semantic recognition using computer vision this may be a list of the imagery that was used to get to this classification, in the case of geometry the log file might link specific point cloud segments to IFC 


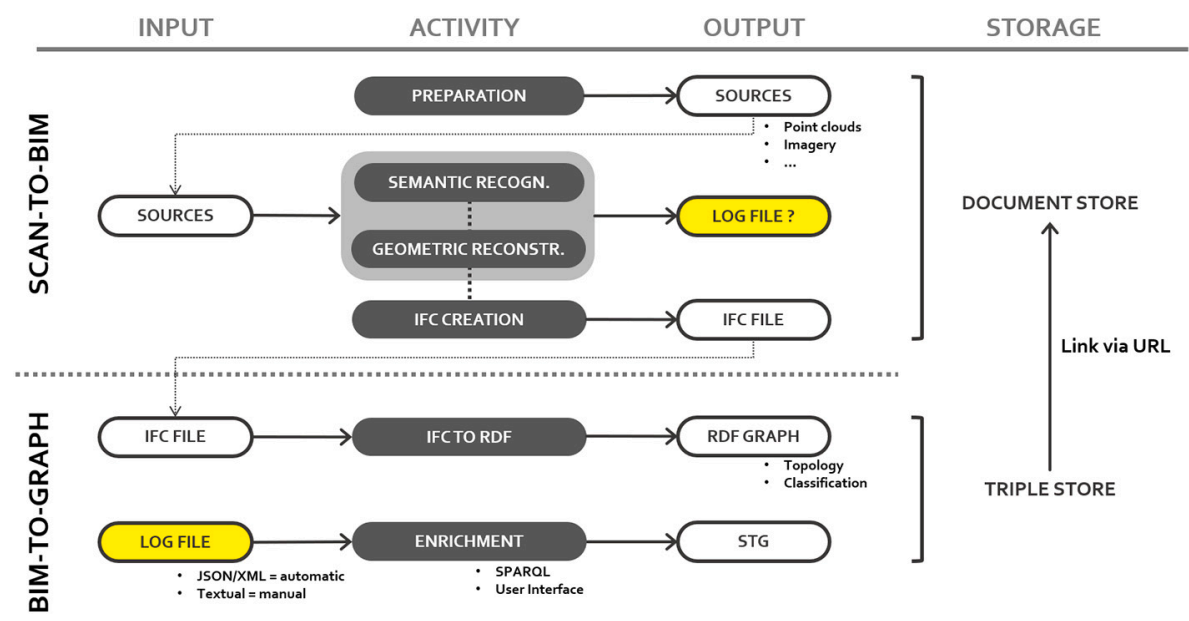

Fig. 5. Using the STG approach from the start of the process. Both the geometric reconstruction process as semantic recognition can be loaded into a triple store as interconnected named graphs.

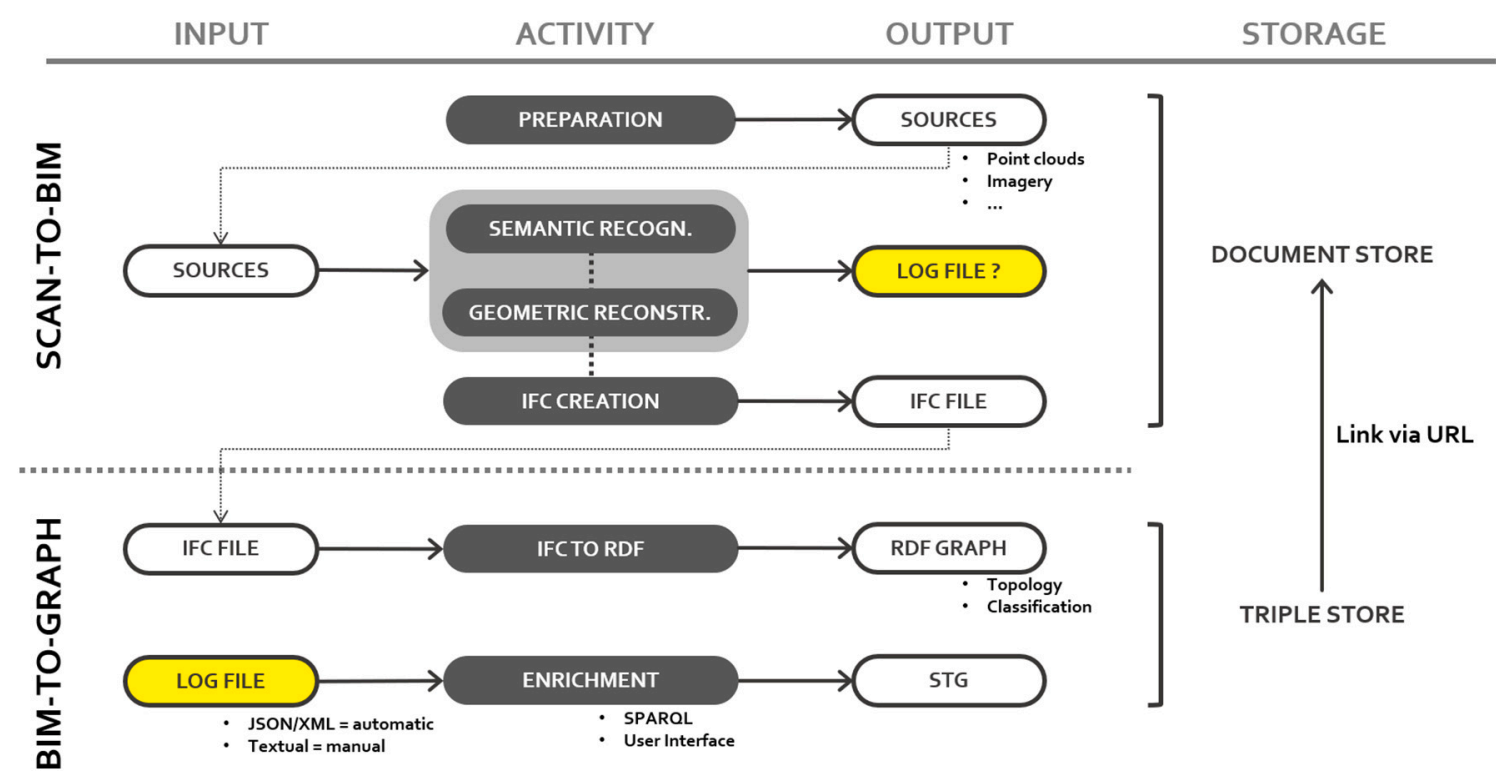

Fig. 6. Extending existing scan-to-BIM solutions with the STG approach. Log files should be serialised in JSON or XML to ease the conversion process in the last step.

GUIDs. As illustrated in Fig. 6, such log file is essential to create the STG-specific links afterwards, i.e. after the IFC file has been converted to RDF. This semantic enrichment then happens either via a SPARQL endpoint that connects to the triple store where the STG graph is stored, or via a dedicated user interface. However, such semantic enrichment will likely be a ponderous task, as these log files will be mostly textbased, difficult to interpret for machines, unless these logs are serialised using widespread formats such as JSON or XML. Even in the latter case, the keys used to refer to specific information will most likely be software specific, so separate templates will be needed for different algorithms. Therefore, the most convenient way, with the largest degree of process automatisation, would be if a scan-to-BIM algorithm offered the choice to export its outcomes to either an RDF or a non-RDF format [40]. Yet this approach will require a much higher effort from the developer side, while the generating of a log file might be a more quicker, short term solution. Storage of generated output during the different phases may take place similar to described above, using either a combination of a file-based system and a triple store, or an implementation of the Linked Data Platform specification. As described in Section 3.2, separate geometric instances will probably not be present in this scenario; instead, it might be contained in the basic IFC file or a purely geometric derivative serialised in, for example, glTF, OBJ or 3DM
(Rhinoceros). If the $\log$ file generated throughout the scan-to-BIM process keeps track of IFC GUIDs, identification of object geometries within a larger geometric file might happen using the workflow indicated in Section 3.2.

\subsection{Separating data and tools}

As stated in Section 2.2, present BIM authoring tools are not always flexible enough regarding the modelling of irregular geometry for every type of building element. Such irregular geometry is found frequently in as-built situations and even more in the case of heritage buildings. Furthermore, the synchronous modelling of semantics and geometry is closely integrated in many current BIM authoring tools: when creating a building element, one has to immediately assign a unique classification for the object, using a built-in taxonomy [29]. For example, how to classify a capital, a column's base or a gargoyle in modern software packages? This way of working can be problematic when such a taxonomy misses a necessary element type. As indicated in Section 3.1, the use of extendable Linked Data ontologies such as BuildingElement addresses this issue. Many architectural offices have their own set of building specifications and element definitions. These can be used flexibly within the proposed context in combination with built-in 
classification systems, standards and widespread vocabularies such as Getty AAT.

Extending on this concept, the STG approach follows recent Web tendencies to separate data from applications, to allow the user to choose the most fit tool for his task: the geometric modelling tool is not necessarily the best tool for enriching the model with metadata, structural or historical information. The application does not manipulate the data directly, but acts as an interface to communicate with the (possibly remotely stored) data. In a general Web context, this approach is devised in the Solid initiative by Tim Berners Lee [41]. In context of construction-related disciplines, this aligns with the goals put forward by the W3C LBD Community Group, and is also related to ambitions within the technical BuildingSMART community to make IFC more Web- and API-based, ${ }^{24}$ or to the use of so-called specialised 'bim bots ${ }^{25}$ that are connected with the BIMserver ecosystem [42].

A requirement in such scenario is to use open source formats and ontologies as much as possible. The RDF data resides in a running triple store and is created, loaded and manipulated at runtime, so multiple applications may connect to this store, serving as an interface to the data rather than 'standalone' applications. Such separation serves the need for services that are fully optimised for their purpose: a tool fit for complex 3D modelling is not necessarily the best tool for semantic recognition of elements, enrichment with historical data or connecting with open data on the web. As a result, partial graphs can be reconstructed in parallel, even by different actors and applications. Information about various subdisciplines, sources or geometric representations can be added freely and on-the-go, using relevant vocabularies and tools that exist on the web.

\section{Implementation: a scan-to-graph environment}

As a proof of concept, a plugin for Rhinoceros 6 was developed to support the creation of an RDF graph according to the STG schema and workflow described in Section 3.5. The plugin code can be found on https://github.com/JWerbrouck/scan-to-graph/tree/master/scan-tograph_Plugin_Rhino6. An interface to visualise geometric results of a SPARQL query executed on a triplestore containing the RDF building model is also included. Although the plugin supports the creation of the graph in Rhinoceros, the resulting graph implements non-proprietary, open geometry formats as much as possible. An exception is the case where geometry is not included in the graph, but only identified by referencing to an external file (such as Rhinoceros files). Under the hood, the tool connects to a Stardog triplestore ${ }^{26}$ and the open source software CloudCompare Stereo. ${ }^{27}$ The tool can easily be extended to work with other RDF triplestores or LDP implementations, as it relies on existing W3C standards. The main functionality of the plugin is focused in four tabs: (1) the Project Info Tab, for stating the geospatial and topological information, (2) the Point Cloud importing Tab, (3) the Semantics Tab, for semantic enrichment of geometries (location, relationships with other elements, product identification and stating metadata remarks) and (4) the SPARQL query Tab for querying the model.

Although the tool described in this chapter is conceived as an aid for manual reconstruction of heritage buildings with complex geometries, theoretically the same degree of automation is possible compared to a regular scan-to-BIM workflow: any recognition algorithm in the scanto-BIM process may produce its outcome as RDF triples as well as its default outcome, hence it can be connected to the triple store and

\footnotetext{
${ }^{24}$ https://github.com/buildingSMART/NextGen-IFC/wiki/Towards-atechnology-independent-IFC.

${ }^{25}$ https://www.tno.nl/en/tno-insights/articles/bim-bots-boost-constructionsector-productivity/.

26 https://www.stardog.com/.

${ }^{27}$ https://www.danielgm.net/cc/.
}

enrich the model with its outcome. Whether a conversion process can be (semi-)automated thus mostly depends on the building of interest: a historic church has a higher chance of having more complex geometries than a modernist villa, so a higher degree of human-computer interaction will be necessary.

\subsection{The project info tab}

In this tab, the overall building topology can be modelled and the location and orientation of the local project origin in a Geographic Coordinate System (GCS) can be defined. First the site and building are defined, followed by the building storeys and the spaces they contain. For now, the exterior of the building is considered a bot:Zone. Adding a Geographic Coordinate System (GCS) for the project is possible by referencing their openGIS URI into the coordinate system input field. Linking multiple GCS within a single project is currently not supported, although there will be no conflicts on ontology level. Georeferenced point clouds that are imported using the plugin will be translated to the local project origin using the project coordinates, if a Cartesian coordinate system is used.

\subsection{The point cloud tab}

When the basic building topology is defined, one can start modelling the as-is geometry based on point clouds (or other sources, e.g. meshes). For importing the point clouds in the E57 file format, the Rhinoceros Plugin 'E57 File Import' by Dale Fugier is used. ${ }^{28}$ The plugin streamlines this importing process for as-is modelling through several steps. The large file size of huge detailed point clouds can result in performance problems in Rhinoceros or other CAD packages. Therefore, subsampling the point clouds by calling CloudCompare prior to their import is given as an option. First of all, each point cloud - ideally one point cloud per building component - is imported and located on a separate layer. Apart from the point cloud, the layer also contains the reconstructed geometry of the corresponding element, in this case the Rhinoceros geometry. In the serialisation phase, this allows to easily bundle all element representations related to the object with omg:hasGeometry.

\subsection{The semantics tab}

This tab constitutes the core of the plugin and is demonstrated in Fig. 7. The 'Main Objects', i.e. larger objects which may have a point cloud representation, refer to a layer of the document.

In the plugin, each building object is located in a space, which is part of a storey, both defined earlier in the 'Project Info' tab. Also, the default relationship between a zone and an object is set to bot:containsElement. The checkbox 'Adjacent' provides the option to override this default relationship and set it as bot:adjacentElement. In this case, the element is no longer contained but serves as the separation between two zones. It then becomes possible to define a bot:Zone on the other side.

An object may have sub-objects (bot:hasSubElement and bpo:consistsof, the inverse property of bpo:isPartOf). An option is provided to set a sub-object as a hosted element of another sub-object, hereby enabling the core Linked Data feature of virtually unlimited detail. For example, an instance of a beo:Column can be further deconstructed into an stgp:Base, an stgp:Shaft and an stgp:Capital instance. In turn, the capital can be subdivided into instances of stgp:Acanthus, stgp:Abacus, etc. Note that, from a certain point, the component classification classes will have to be custom-defined, since they will not be included in the BuildingElement or other ontologies anymore. As indicated in Section 3.1 and Table 2, these custom definitions may refer to existing

\footnotetext{
${ }^{28}$ https://www.food4rhino.com/app/e57-file-import.
} 

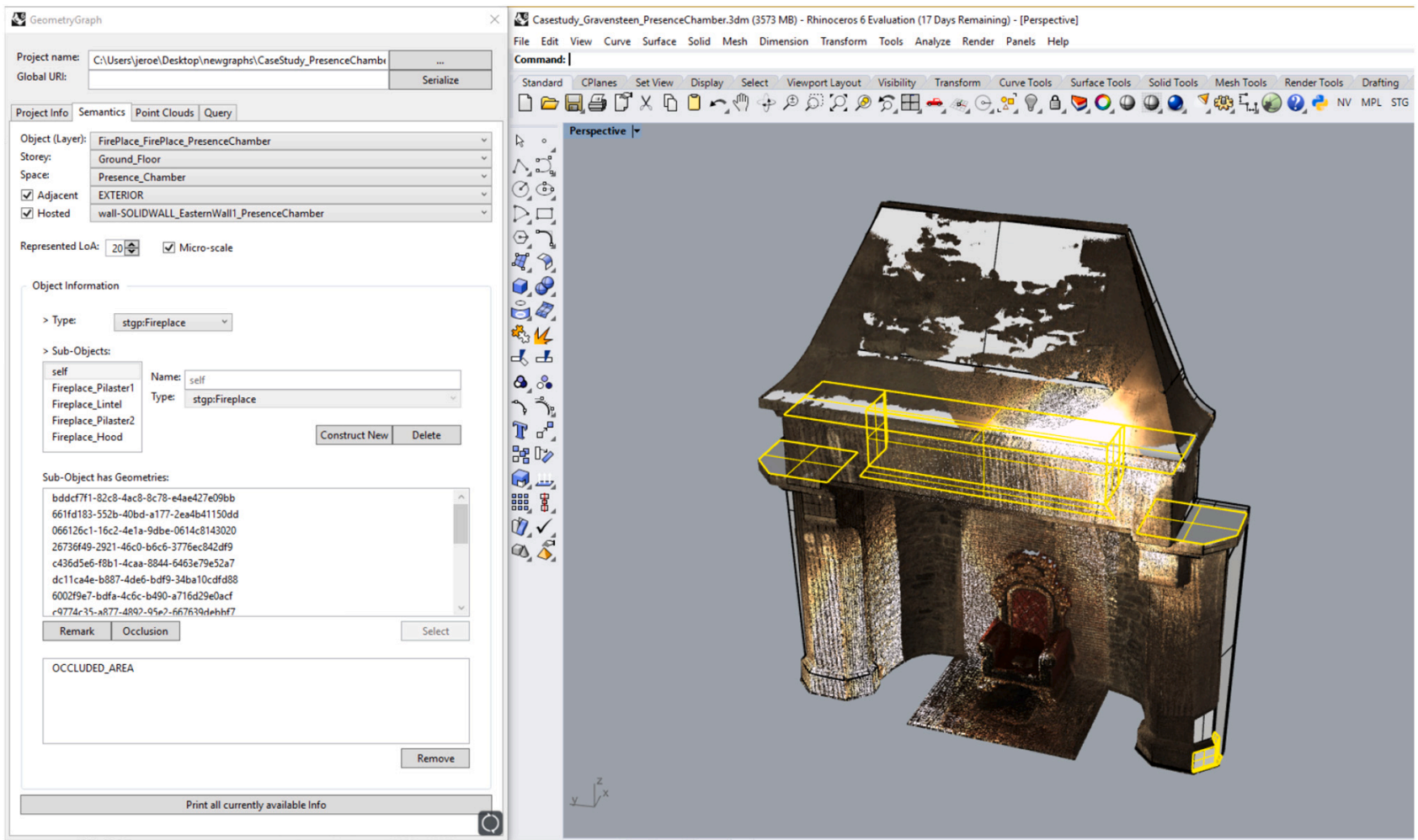

Fig. 7. Interface of the semantics tab in relation to an as-is model of the case study in Rhinoceros 6 . The interface allows to label the highlighted reconstructed surfaces as stg:Occlusion.

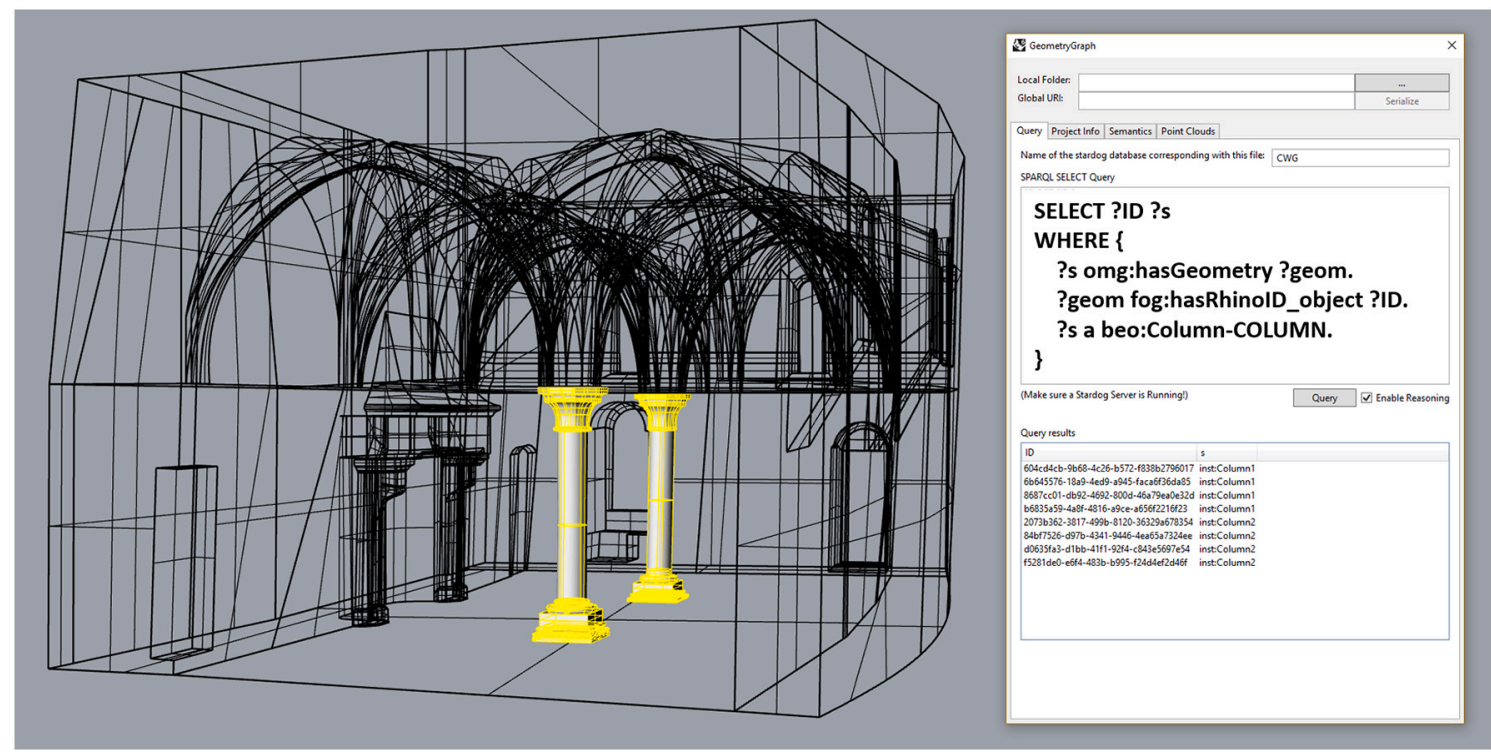

Fig. 8. Interface of the SPARQL query tab. Query results, in this case all geometries belonging to a beo:Column-COLUMN instance, are highlighted in the viewer. The SPARQL query example is enlarged for better readability.

definitions in other vocabularies by an rdfs:seeAlso statement.

In the plugin, each object intrinsically aggregates the 3D geometry that its corresponding layer contains. Selecting an element makes it possible to add one or more written notes to this semantic element in the form of an assumption (stg:Assumption). It is also possible to directly relate these to an occluded geometry (stg:Occlusion), in the plugin both listed in the lowest section of the tab. Following a deviation analysis, the resulting Level of Accuracy can be linked to the respective element.

\subsection{The SPARQL query tab}

The last tab, depicted in Fig. 8, is used for executing SPARQL queries and visualising geometry that matches the query results. The $\mathrm{RDF}$ graph needs to be loaded in a running triplestore before running a query. The example query searches to visualise Rhino IDs, related to the larger '. $3 \mathrm{dm}$ ' (Rhino) file following the methodology described in Section 3.2. The graph structure depicted in Fig. 3 is used to find the object within the larger 3DM document. When the query yields any results, the overall display mode changes to 'wireframe', highlighting 
objects related to the found IDs. The example query in Fig. 8 visualises all Rhino-geometries that are part of a beo:Column-COLUMN instance.

\section{Case study: the Gravensteen Castle}

As a proof of concept, a geometric model of the Audience Room in the Gravensteen Castle in Ghent has been modelled in Rhinoceros, based on point clouds. The point clouds were based on the results from photogrammetric and terrestrial laser scanning surveys. In line with the Research Question in Section 1.3, the aim is to construct a modular RDF graph of the existing asset, which (1) contains a basic semantic model of the building, (2) contains metadata about the reconstruction process and (3) can be further extended in a future phase using other Semantic Web ontologies (e.g. to link historical events to building elements) or public data on the Web.

The preparation phase included alignment of the point cloud data, georeferencing and manual clutter removal (post-processing of point cloud sources). To enable fine-grained linking of building objects to point clouds, and to streamline the usage of the point clouds in the CAD modelling environment, the aligned point clouds were segmented per building element.

In general, creating the semantic model, which consists of the building topology, product classification and hierarchy, can happen independently from the geometric modelling [29]. Based on existing source documents, one can start defining the building's topology. However, as the plugin uses the point clouds as indication for the building elements present in the model, the semantic modelling step could benefit from having the point clouds imported in the environment.

In the case of heritage buildings, the building geometry can be very irregular and thus difficult to accurately reconstruct as NURBS or BREP geometry. Although surfaces of objects can be reconstructed quite well by existing algorithms, the 'invisible' parts such as the internal boundaries, occlusions etc. remain a limitation when existing algorithms are used for reconstruction. Therefore, human decision making is still essential in this process. In the presented use case, mostly manual modelling techniques were used, the segmented point clouds serving as an underlay. As indicated in Section 4.2, the plugin uses object layers to automatically link imported sources and reconstructed geometries to one and the same semantic object. A general Level of Accuracy of LOA20, corresponding to a maximum deviation of $15 \mathrm{~mm}$, was set as a modelling requirement. After modelling an element, the reconstructed geometry was compared to the underlying point cloud during a deviation analysis, using the techniques for macro and micro analysis as described in [26].

In the next phase, the building object was semantically enriched with the plugin. An example is given in Fig. 9, depicting the reconstructed geometry of the fireplace in the Audience Room. In this example, the storey and space of the fireplace are set to 'Ground_Floor' and 'Audience_Room'. The fireplace is hosted (bot:hasSubElement) by the Eastern wall of the room. The fireplace object is classified as stgp:Fireplace and consists of four sub-objects, each one with an internal and an external (visible) part. The BPO ontology is used to describe part-whole relationships (bpo:isPartOf):

\section{Fireplace_Pilaster1 (beo:Column-PILASTER); \\ 2. Fireplace_Pilaster2 (beo:Column-PILASTER); \\ 3. Fireplace_Lintel (beo:Beam-LINTEL); \\ 4. Fireplace_Hood (stgp:Fireplace_Hood)}

Each of these sub-objects and surrounding components are subject to specific uncertainties. During the modelling phase, the following assumptions were made:

1. The height of the restored chimney (occluded area) is equal to the height of the hood. This was annotated as an rdfs:comment linked to an stg:Assumption instance. The occluded geometries were labelled accordingly using stg:Occlusion. As depicted in Fig. 7, there are several surfaces in the geometric model that are not covered by the point cloud, which are labelled as stg:Occlusion as well.

2. Part of the base of the right pilaster was occluded by furniture at the time of the surveys. It was therefore modelled by interpolating visible edges. This information can be linked to the geometric object node in the form of a literal.

3. The chimney depth was set equal to the depth of the 'lintel', also by interpolation of visible edges.

4. Also, the other 'inside' parts of the elements (enclosed by the wall) were separated from the outside parts, forming different volumes (Fig. 9). The stg:isInternalPartOf relationship is used to denote that this geometry is reconstructed with high uncertainty.

This process of linking assumptions and remarks to a geometric representation of a model can be applied to any geometric instance, whether to identify a single surface, part of a larger object, as being an stg:Occlusion (Fig. 7) or to take general notes during the reconstruction process. With this in mind, a detailed record of modelling choices can be directly linked to the objects of interest.

The case-study briefly illustrates the workflow of the proposed scanto-graph process. The first part of the process does not differ from a regular scan-to-BIM process: the available point clouds were segmented as a preparation for the $3 \mathrm{D}$ modelling phase. Next, comparing the $3 \mathrm{D}$ reconstructions (manually or using a scan-to-BIM plugin) of the elements with the source point clouds is an essential feedback step for any as-is geometry reconstruction method. The final phase in the scan-tograph workflow is different from regular scan-to-BIM processes, as it involves the semantic enrichment of the geometry by creating an RDF graph. This includes the modelling of the building topology, element classification and modelling of metadata related to occlusions, modelling remarks and assumptions. After the basic model is made, RDFbased information is stored in a triple store, while non-RDF data (point clouds, geometry) are stored in a file-based system (Section 3.5).

\section{Evaluation and future work}

\subsection{Evaluation}

In this research, an approach complementary to present scan-to-BIM workflows was developed. In line with the original research question (Section 1.3), we developed a workflow to create an extendable model of a built asset. To come to this workflow, we investigated how Linked Data techniques can be used to cope with several known challenges that prevent a more frequent application of as-is BIM models for existing buildings. This included, apart from identifying relevant ontologies to consolidate the approach, an interpretation of the scan-to-BIM process in a Linked Data context, which was named 'scan-to-graph'. A novel STG vocabulary was devised to facilitate this process. Furthermore, the conditions for extending a regular scan-to-BIM process to incorporate Linked Data technologies were shown; essentially only accurate logging of the conversion and recognition process is required to afterwards incorporate them into the overall graph of the model (Section 3.5). Essentially, the scan-to-graph workflow addresses the following topics:

1. Linking various representations: Multiple source and reconstruction resources can be referenced and linked to one another in the same knowledge graph. Non-RDF data such as point clouds, imagery and multiple geometry formats are related to the same semantic model.

2. Keeping track of assumptions: As there are always uncertainties in the available information, keeping track of modelling assumptions is a vital part of the reconstruction process. Different types of uncertainties can be distinguished and documented as such, relating the reconstruction to the source.

3. Facilitate an interdisciplinary approach: Managing heritage assets is a 


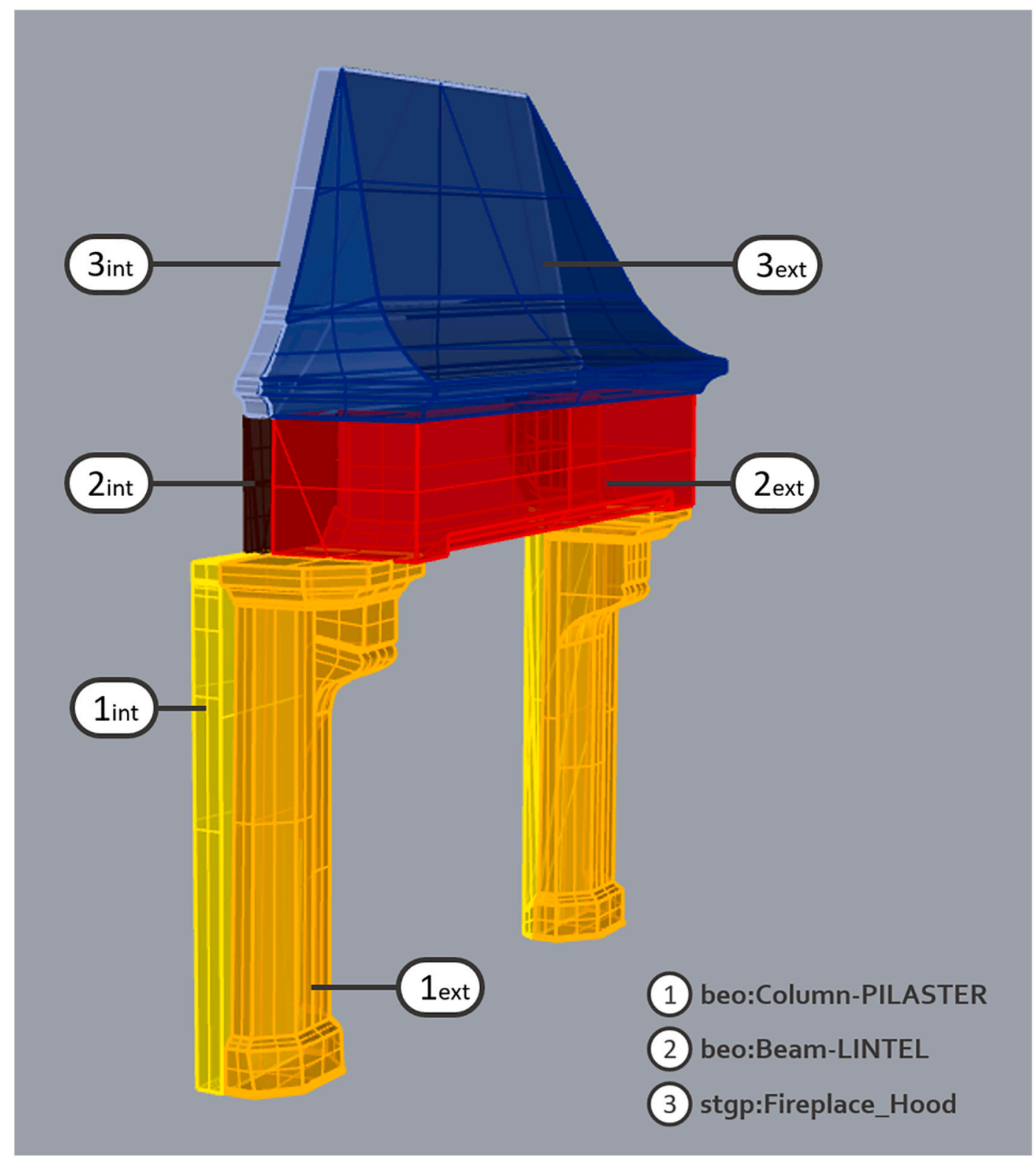

Fig. 9. Different volumes form the fireplace. Geometries that are enclosed by the wall are assumed, which is indicated by a part-whole relationship stg:isInternalPartOf (see also Fig. 2).

multidisciplinary activity, where many actors with many backgrounds collaborate. Migrating this practice to the discipline-independent Semantic Web allows all actors to use a standardised data representation framework, RDF. The use of Web-based data standards also allows separating this data from applications: specialised applications can offer discipline-specific perspectives on the same datasets. Geometric modeller, software agents for semantic recognition and linking to contextual or historic data all require such different viewpoints, to maximise the flexibility for adding semantics, sources and geometries. Apart from ontological data from multiple domains, this includes non-RDF resources such as geometry and imagery on the web as well.

The combination of product data with building data is mentioned in [17] in the case of product manufacturer data. In the context of as-is buildings, this can be broadened to also include historical building components. As an illustration, the building element classification classes that were developed for specific elements in the case study are an addition to the BuildingElement Ontology and also link to RDF- based definitions in the Art and Architecture Thesaurus (AAT) and Wikidata. Extra information can be retrieved by dereferencing the URIs related to those definitions. This ability to classify non-common elements may sometimes be seen as a drawback, since it is possible that users start creating their own classes, without checking if existing (standardised) taxonomies already include similar entities. To minimise this risk, STG applications should therefore allow easy querying of those existing taxonomies, e.g. by directly connecting to APIs exposing those taxonomies or by using ontology crawlers such as https://lov. linkeddata.es. In this way, the user can be presented existing solutions, which might be used for a custom extension only if necessary.

Another challenge is the high threshold for developing Linked Datacompatible applications. Although efforts are taken to lower this threshold, ${ }^{29}$ information on how to develop such tools is not yet widespread. This may also hold back developers of existing scan-to-BIM

\footnotetext{
${ }^{29}$ https://ruben.verborgh.org/blog/2018/12/28/designing-a-linked-datadeveloper-experience/.
} 
applications to implement a hybrid approach in which the tool is able to export RDF-based results as well. In Section 3.5 it was indicated that log files of scan-to-BIM applications may be used to enrich the graph in a later phase, but it may be clear that this is not the optimal solution, since those log files will often consist of textual logs without any semantic meaning attached to it. Re-interpretation of this data to be able to link it to the RDF model will therefore be a cumbersome task. The inclusion of $\log$ files in existing algorithms is therefore a more shortterm strategy, easing the alignment with the STG workflow but at the end requiring higher conversion effort. It is therefore recommended to either offer the choice to export the output to RDF immediately, or, when this is an effort not feasible from the developer's perspective, to serialize the $\log$ file into a more widespread computer-readable format such as JSON or XML, in order to ease the conversion to RDF afterwards (Fig. 6).

\subsection{Future work}

In the current version of STG, the metadata terminology is limited to assumptions, occlusions, stating that a geometry is 'internal' and indicating deviations between reconstruction and source. In future research, sub-classes specific for other types of assumptions or uncertainties related to properties need to be developed. Also, the STG vocabulary in general will be further assessed in different environments and the potential to link the minimal BIM model to data originating from other disciplines such as sensor data, FM, heritage conservation and GIS will be explored further. In terms of practical implementation, future versions of the semantic enrichment plugin will move towards a web-based environment and further investigate alignment with existing scan-to-BIM workflows and algorithms, to lower the threshold for following an STG workflow starting from a regular scan-to-BIM process. Finally, to support a possible transition from a scan-to-BIM environment to scan-to-Graph, automatic interpretation of generated log files from existing algorithms will be investigated.

\section{Conclusion}

The presented research demonstrates a framework to interlink available information about an existing building: its topology, source data, geometry of individual elements and related modelling remarks. The proposed scan-to-graph framework builds upon work by other researchers in the Linked Building Data Community and adds the specific scenario of documenting scan-to-BIM processes using Linked Data. Using the flexibility of Web technologies, a workflow is initiated that can be part of a variety of existing building scenarios.

\section{Declaration of competing interest}

The authors declare that they have no known competing financial interests or personal relationships that could have appeared to influence the work reported in this paper.

\section{Acknowledgements}

This research is funded by the Research Foundation Flanders (FWO) in the form of a grant Strategic Basic Research (grant no. 1S99020N and $1 \mathrm{~S} 65917 \mathrm{~N})$. We would also like to thank the KU Leuven Geomatics research group (Ghent) for providing the point clouds used in this research, and acknowledge the support provided by the BIM4Ren project, which has received funding from the European Union's Horizon 2020 Research and Innovation Program under grant agreement No 820773.

\section{Appendix A. Supplementary data}

Supplementary data to this article can be found online at https:// doi.org/10.1016/j.autcon.2020.103286.

\section{References}

[1] C. Eastman, P. Teicholz, R. Sacks, K. Liston, BIM Handbook: A Guide to Building Information Modeling for Owners, Managers, Designers, Engineers and Contractors, 2nd edition, John Wiley \& Sons, Hoboken, New York, United States, 2011.

[2] A. Borrmann, M. König, C. Koch, J. Beetz, Building Information Modeling: Why? What? How? Springer International Publishing, 978-3-319-92862-3, 2018, pp. 1-24, https://doi.org/10.1007/978-3-319-92862-3_1 (Ch. 1).

[3] R. Volk, J. Stengel, F. Schultmann, Building information modeling (BIM) for existing buildings - literature review and future needs, Autom. Constr. 38 (2014) 109-127, https://doi.org/10.1016/j.autcon.2013.10.023.

[4] M. Barbosa, P. Pauwels, V. Ferreira, L. Mateus, Towards increased BIM usage for existing building interventions, Struct. Surv. 34 (2) (2016) 168-190, https://doi. org/10.1108/SS-01-2015-0002.

[5] S. Bruno, M. De Fino, F. Fatiguso, Historic building information modelling: performance assessment for diagnosis-aided information modelling and management, Autom. Constr. 86 (2018) 256-276, https://doi.org/10.1016/j.autcon.2017.11. 009.

[6] Y. Shi, P. Long, K. Xu, H. Huang, Y. Xiong, Data-driven contextual modeling for 3d scene understanding, Comput. Graph. 55 (2016) 55-67, https://doi.org/10.1016/j. cag.2015.11.003.

[7] S. Ochmann, R. Vock, R. Wessel, R. Klein, Automatic reconstruction of parametric building models from indoor point clouds, Comput. Graph. 54 (2016) 94-103, https://doi.org/10.1016/j.cag.2015.07.008.

[8] M. Bassier, M. Vergauwen, B. Van Genechten, Automated classification of heritage buildings for as-built BIM using machine learning techniques, ISPRS Annals of the Photogrammetry, Remote Sensing and Spatial Information Sciences, IV-2/W2 2017, pp. 25-30, , https://doi.org/10.5194/isprs-annals-IV-2-W2-25-2017 Ottawa, Canada.

[9] C. Thomson, From Point Cloud to Building Information Model: Capturing and Processing Survey Data Towards Automation for High Quality 3D Models to Aid a BIM Process (Ph.D. thesis), UCL (University College London), 2016 URL https:// discovery.ucl.ac.uk/id/eprint/1485847/.

[10] D. Mezzino, Cultural Built Heritage's Tangible and Intangible Dimensions and Digitalization Challenges (Ph.D. thesis), Carleton University, 2017 URL, https:// curve.carleton.ca/93b099c7-a394-48eb-8ed7-0be74eb58837.

[11] M.K. Dixit, V. Venkatraj, M. Ostadalimakhmalbaf, F. Pariafsai, S. Lavy, Integration of facility management and building information modeling (BIM), Facilities 37 (2019) 455-483, https://doi.org/10.1108/F-03-2018-0043.

[12] P. Pishdad-Bozorgi, X. Gao, C. Eastman, A.P. Self, Planning and developing facility management-enabled building information model (FM-enabled BIM), Autom. Constr. 87 (2018) 22-38, https://doi.org/10.1016/j.autcon.2017.12.004.

[13] T. Berners-Lee, J. Hendler, O. Lassila, et al., Semant. Web Sci. Am. 284 (5) (2001) 28-37, https://doi.org/10.1038/scientificamerican0501-34.

[14] J. Beetz, J. van Leeuwen, B. de Vries, An ontology web language notation of the industry foundation classes, in: R. Scherer, P. Katranuschkov, S.-E. Sconfke (Eds.), Proceedings of the 22nd CIB W78 Conference on Information Technology in Construction, Technische Universität Dresden, 2005, pp. 193-198.

[15] P. Pauwels, W. Terkaj, EXPRESS to OWL for construction industry: towards a recommendable and usable ifcOWL ontology, Autom. Constr. 63 (2016) 100-133, https://doi.org/10.1016/j.autcon.2015.12.003.

[16] M.H. Rasmussen, P. Pauwels, M. Lefrançois, G.F. Schneider, C.A. Hviid, J. Karlshøj, Recent changes in the building topology ontology, 5th Linked Data in Architecture and Construction Workshop (LDAC), Dijon, France, 2017, https://doi.org/10. 13140/RG.2.2.32365.28647 URL https://hal-emse.ccsd.cnrs.fr/emse-01638305.

[17] P. Pauwels, S. Zhang, Y.-C. Lee, Semantic web technologies in AEC industry: a literature overview, Autom. Constr. 73 (2017) 145-165, https://doi.org/10.1016/j. autcon.2016.10.003.

[18] P. Grussenmeyer, T. Landes, T. Voegtle, K. Ringle, Comp arison methods of terrestrial laser scanning, photogrammetry and tacheometry data for recording of cultural heritage buildings, Int. Arch. Photogramm. Remote. Sens. Spat. Inf. Sci. 37 (B5) (2008) 213-218 URL https://www.isprs.org/proceedings/XXXVII/congress/5_ pdf/38.pdf.

[19] C. Brenner, Building reconstruction from images and laser scanning, Int. J. Appl. Earth Obs. Geoinf. 6 (3-4) (2005) 187-198, https://doi.org/10.1016/j.jag.2004.10. 006.

[20] M. Bassier, M. Bonduel, B. Van Genechten, M. Vergauwen, Segmentation of large unstructured point clouds using octree-based region growing and conditional random fields, Int. Arch. Photogramm. Remote. Sens. Spat. Inf. Sci. 42 (2W8) (2017) 25-30, https://doi.org/10.5194/isprs-archives-XLII-2-W8-25-2017.

[21] H. Son, C. Kim, Semantic as-built 3d modeling of structural elements of buildings based on local concavity and convexity, Adv. Eng. Inform. 34 (2017) 114-124, https://doi.org/10.1016/j.aei.2017.10.001.

[22] S. Ochmann, R. Vock, R. Klein, Automatic reconstruction of fully volumetric 3d building models from oriented point clouds, ISPRS J. Photogramm. Remote Sens. 151 (2019) 251-262, https://doi.org/10.1016/j.isprsjprs.2019.03.017.

[23] J. Chen, Y. Fang, Y.K. Cho, Performance evaluation of 3D descriptors for object recognition in construction applications, Autom. Constr. 86 (2018) 44-52, https:// doi.org/10.1016/j.autcon.2017.10.033.

[24] R.J. Scherer, P. Katranuschkov, Bimification: how to create and use BIM for retrofitting, Adv. Eng. Inform. 38 (2018) 54-66, https://doi.org/10.1016/j.aei.2018. 05.007.

[25] U. S. Institute of Building Documentation, USIBD Level of Accuracy (LOA) 
Specification Guide, Tech. Rep, U.S. Institute of Building Documentation, 2016 URL, https://usibd.org/product/level-of-accuracy-loa-specification-version-2-0/.

[26] M. Bonduel, M. Bassier, M. Vergauwen, P. Pauwels, R. Klein, Scan-to-BIM output validation: towards a standardized geometric quality assessment of building information models based on point clouds, ISPRS - International Archives of the Photogrammetry, Remote Sensing and Spatial Information Sciences, Vol. XLII-2/ W8, Hamburg, Germany, 2017, pp. 45-52, , https://doi.org/10.5194/isprsarchives-XLII-2-W8-45-2017.

[27] G.F. Schneider, M.H. Rasmussen, P. Bonsma, J. Oraskari, P. Pauwels, Linked building data for modular building information modelling of a smart home, 12th European Conference on Product and Process Modelling (ECPPM), CRC Press, Copenhagen, Denmark, 2018, pp. 407-414, , https://doi.org/10.1201/ 9780429506215-51.

[28] M. Bonduel, J. Oraskari, P. Pauwels, M. Vergauwen, R. Klein, The IFC to linked building data converter - current status, 6th Linked Data in Architecture and Construction Workshop (LDAC), CEUR Workshop Proceedings, 2159 2018, pp. 34-43 URL http://ceur-ws.org/Vol-2159/04paper.pdf (London, United Kingdom).

[29] M. Bonduel, M.H. Rasmussen, P. Pauwels, M. Vergauwen, R. Klein, A novel workflow to combine BIM and linked data for existing buildings, 12th European Conference on Product and Process Modelling (ECPPM), CRC Press, Copenhagen, Denmark, 2018, pp. 347-354, , https://doi.org/10.1201/9780429506215-43.

[30] D. Simeone, S. Cursi, M. Acierno, BIM semantic-enrichment for built heritage representation, Autom. Constr. 97 (2019) 122-137, https://doi.org/10.1016/j. autcon.2018.11.004.

[31] A. Wagner, U. Rüppel, Bpo: The building product ontology for assembled products, 7th Linked Data in Architecture and Construction Workshop (LDAC), CEUR Workshop Proceedings, 2019, pp. 106-119 URL http://ceur-ws.org/Vol-2389/ 08paper.pdf.

[32] M. Bonduel, A. Wagner, P. Pauwels, M. Vergauwen, R. Klein, Including widespread geometry schemas into linked data based BIM applied to built heritage, Proc. Inst. Civ. Eng. Smart Infrastruct. Constr. (2020) 1-16, https://doi.org/10.1680/jsmic.19. 00014.

[33] A. Wagner, M. Bonduel, P. Pauwels, U. Rüppel, Relating geometry descriptions to its derivatives on the web, Proceedings of the European Conference on Computing in
Construction (EC3 2019), Chania, Greece, 2019, pp. 304-313, , https://doi.org/10. 35490/EC3.2019.146.

[34] M. Bonduel, A. Wagner, P. Pauwels, M. Vergauwen, R. Klein, Including widespread geometry formats in semantic graphs using RDF literals, Proceedings of the European Conference on Computing in Construction (EC3 2019), Chania, Greece, 2019, pp. 341-350, , https://doi.org/10.35490/EC3.2019.166.

[35] P. Pauwels, T. Krijnen, W. Terkaj, J. Beetz, Enhancing the ifcOWL ontology with an alternative representation for geometric data, Autom. Constr. 80 (2017) 77-94, https://doi.org/10.1016/j.autcon.2017.03.001.

[36] K. McGlinn, A. Wagner, P. Pauwels, P. Bonsma, P. Kelly, D. O‘Sullivan, Interlinking geospatial and building geometry with existing and developing standards on the web, Autom. Constr. 103 (2019) 235-250, https://doi.org/10.1016/j.autcon.2018. 12.026 .

[37] A.-H. Hamdan, M. Bonduel, R.J. Scherer, An ontological model for the representation of damage to constructions, 7th Linked Data in Architecture and Construction Workshop (LDAC), CEUR Workshop Proceedings, 2019, pp. 64-77 URL http://ceur-ws.org/Vol-2389/05paper.pdf.

[38] M.H. Rasmussen, M. Lefrançois, P. Pauwels, C.A. Hviid, J. Karlshøj, Managing interrelated project information in AEC Knowledge Graphs, Autom. Constr. 108 (2019), https://doi.org/10.1016/j.autcon.2019.102956.

[39] M. Rasmussen, M. Lefrançois, M. Bonduel, C.A. Hviid, J. Karlshøj, Opm: an ontology for describing properties that evolve over time, 6th Linked Data in Architecture and Construction Workshop (LDAC), CEUR Workshop Proceedings, 2159 2018, pp. 24-33 URL http://ceur-ws.org/Vol-2159/03paper.pdf.

[40] M. Bassier, M. Bonduel, J. Derdaele, M. Vergauwen, Processing existing building geometry for reuse as linked data, Autom. Constr. 115 (2020) 103180, , https://doi. org $/ 10.1016 /$ j.autcon.2020.103180.

[41] E. Mansour, A.V. Sambra, S. Hawke, M. Zereba, S. Capadisli, A. Ghanem, A. Aboulnaga, T. Berners-Lee, A demonstration of the solid platform for social web applications, Proceedings of the 25th International Conference Companion on World Wide Web, 2016, pp. 223-226, , https://doi.org/10.1145/2872518. 2890529.

[42] J. Beetz, L. van Berlo, R. de Laat, P. van den Helm, Bimserver.org-an open source ifc model server, Proceedings of the CIP W78 Conference, 2010, p. 8. 